\title{
Obesidade: diagnóstico e tratamento farmacológico com Liraglutida, integrado a terapia comportamental e mudanças no estilo de vida
}

\author{
Obesity: diagnosis and pharmacological treatment with Liraglutide, integrated with \\ behavioral therapy and changes in lifestyle
}

Obesidad: diagnóstico y tratamiento farmacológico con Liraglutida, integrado a la terapia conductual y cambios en el estilo de vida.

Layandra Vittória de Assis ${ }^{1 *}$, Amanda Caroline da Silva Morais ${ }^{2}$, Isadora Schwartz Meireles ${ }^{3}$, Ludmilla Ferreira da Costa ${ }^{4}$, Maria Luíza Alves Guerra ${ }^{1}$, Maria Vitória Guerini Novaes ${ }^{3}$, Thaís Cunha Aguiar Gomes ${ }^{5}$, Virgínia Modenesi ${ }^{3}$, Yves Henrique Faria Dias ${ }^{6}$, Rachel Cardoso Lopes Rêgo ${ }^{7}$.

\begin{abstract}
RESUMO
Objetivo: Discorrer sobre a integração da Terapia Comportamental Intensiva para Obesidade (IBT) em conjunto com a farmacoterapia (Liraglutida) e mudanças no estilo de vida para o tratamento de pacientes obesos. Revisão bibliográfica: A obesidade é uma doença inflamatória crônica que teve um aumento significativo nos últimos anos e que representa um fator de risco aumentado para diabetes tipo 2, doenças cardiovasculares, câncer e apneia do sono. $O$ tratamento da obesidade deve ser realizado de forma individual e multidisciplinar, integrando a IBT com a farmacoterapia, em casos de IMC $>30 \mathrm{~kg} / \mathrm{m}^{2}$ ou com IMC>27kg/m² com comorbidade associada. Dentre os fármacos análogos ao GLP-1 existentes, a liraglutida é uma das substâncias mais conhecidas e amplamente difundidas em associação com a IBT, dobrando a média de perda de peso $(11,5 \%)$ em pacientes obesos a curto prazo e, quando incluídas a readequação alimentar e exercícios físicos, foram observadas melhoras a longo prazo. Considerações finais: A Liraglutida é um importante medicamento para induzir e manter a perda de peso em pacientes obesos, contudo é um medicamento de aplicação subcutânea e de alto custo.
\end{abstract}

Palavras-chave: Liraglutida, Obesidade, Farmacologia.

\begin{abstract}
Objective: To discuss the integration of Intensive Behavioral Therapy for Obesity (IBT) in conjunction with pharmacotherapy (Liraglutide) and changes in lifestyle for the treatment of obese patients. Bibliographic review: Obesity is a chronic inflammatory disease that has increased significantly in recent years and represents an increased risk factor for type 2 diabetes, cardiovascular disease, cancer and sleep apnea. The treatment of obesity must be carried out individually and in a multidisciplinary way, integrating IBT with pharmacotherapy, in cases of $\mathrm{BMl}>30 \mathrm{~kg} / \mathrm{m}^{2}$ or with $\mathrm{BMl}>27 \mathrm{~kg} / \mathrm{m}^{2}$ with associated comorbidity. Amongthe existing drugs similar to GLP-1, liraglutide is one of the most well-known and widespread substances in association with IBT, doubling the average weight loss (11.5\%) in obese patients in the short term and when including food readjustment and exercise, long-term improvements were observed. Final considerations Liraglutide is an important drug to maintain and induce weight loss in obese patients, however it is a subcutaneous and expensive drug.
\end{abstract}

Keywords: Liraglutide, Obesity, Pharmacology.

${ }^{1}$ Instituto Metropolitano de Ensino Superior do Vale do Aço (IMES UNIVAÇO), Ipatinga - MG.

*E-mail: layandraassis@yahoo.com.br

2 Universidade de Rio Verde (UniRV), Aparecida de Goiânia - GO.

3 Universidade de Vila Velha (UVV), Vila Velha - ES.

${ }^{4}$ Universidade do Sul de Santa Catarina (UNISUL), Tubarão - SC.

${ }^{5}$ Pontifícia Universidade Católica de Goiás (PUC-GO), Goiânia - GO.

${ }^{6}$ Faculdade de Ciências Médicas e da Saúde de Juiz de Fora (FCMS/JF), Juiz de Fora - MG.

${ }^{7}$ Faculdade Ciências Médicas de Minas Gerais (FCMMG), Belo Horizonte - MG.

SUBMETIDO EM: 2/2021

ACEITO EM: 3/2021

PUBLICADO EM: 5/2021 


\section{RESUMEN}

Objetivo: Discutir la integración de la Terapia Conductual Intensiva para la Obesidad (TBI) junto con la farmacoterapia (liraglutida) y los cambios en el estilo de vida para el tratamiento de pacientes obesos. Revisión bibliográfica: La obesidad es una enfermedad inflamatoria crónica que ha aumentado significativamente en los últimos años y representa un mayor factor de riesgo de diabetes tipo 2, enfermedad cardiovascular, cáncer y apnea del sueño. El tratamiento de la obesidad debe realizarse de forma individual y multidisciplinar, integrando el TBI con la farmacoterapia, en los casos de IMC> $30 \mathrm{~kg} / \mathrm{m}^{2}$, o con IMC> 27 $\mathrm{kg} / \mathrm{m}^{2}$ con comorbilidad asociada. Entre los fármacos existentes similares al GLP-1, la liraglutida es una de las sustancias más conocidas y extendidas en asociación con IBT, duplicando la pérdida de peso promedio $(11,5 \%)$ en pacientes obesos a corto plazo y al incluir reajuste de alimentos y ejercicio, se observaron mejoras a largo plazo. Consideraciones finales: la liraglutida es un fármaco importante para mantener e inducir la pérdida de peso en pacientes obesos, sin embargo es un fármaco subcutáneo y costoso.

Palabras clave: Liraglutida, Obesidad, Farmacología.

\section{INTRODUÇÃO}

A obesidade é uma doença crônica prevalente, complexa, progressiva, recidivante, de etiologia multifatorial e caracteriza-se por gordura corporal anormal ou excessiva, com prejuízos à saúde. Resulta de diversos fatores complexos como genética, ambiente, estilos de vida e fatores emocionais, abordados no Foresight Obesity Systems Map que apresenta 108 variáveis, conectadas por aproximadamente 300 ligações causais (WHARTON MD, et al., 2020; VANDENBROECK P, et al., 2007; MCGLASHAN J, et al., 2018).

O aumento crescente do número de obesos no mundo indica grande participação do ambiente na gênese da doença, com associação de hábitos dietéticos, sedentarismo e fatores psicossociais, responsáveis por $95 \%$ dos casos. O ambiente e o estilo de vida moderno são potentes estímulos para obesidade, visto que há aumento do consumo de alimentos industrializados, redução da prática de atividade física, alteração do ritmo circadiano que provoca alterações hormonais e aumento dos níveis de estresse que interferem no hábito alimentar. Além disso, cerca de $5 \%$ dos pacientes obesos exibirão alguma causa identificada, sendo $2 \%$ associados a síndromes genéticas raras, o restante, a causas endócrinas e secundárias a medicamentos (MORETZSOHN M, et al., 2015; ABESO, 2016).

Em 2025, a estimativa é de que 2,3 bilhões de adultos no mundo estejam acima do peso, sendo 700 milhões de indivíduos com obesidade (Índice de Massa Corporal (IMC) $\geq 30 \mathrm{~kg} / \mathrm{m}^{2}$ ). No Brasil a obesidade aumentou $67,8 \%$ nos últimos treze anos, a maior taxa de crescimento foi entre adultos de 25 a 34 anos $(84,2 \%)$ e de 35 a 44 anos (81,1\%) (ABESO, 2016).

De acordo com os dados da Pesquisa de Vigilância de Fatores de Risco e Proteção para Doenças Crônicas por Inquérito Telefônico, que é realizada anualmente pelo Ministério da Saúde no Brasil, em 2019, no conjunto das 27 cidades avaliadas, a frequência de excesso de peso (IMC $\left.\geq 25 \mathrm{~kg} / \mathrm{m}^{2}\right)$ foi de $55,4 \%$, sendo ligeiramente maior entre homens $(57,1 \%)$ do que entre mulheres $(53,9 \%)$. Entre homens, a frequência dessa condição aumentou com a idade até os 44 anos e com baixa escolaridade. Entre as mulheres, a frequência do excesso de peso aumentou com a idade até os 64 anos e diminuiu notavelmente com o aumento da escolaridade. A frequência de adultos obesos (IMC $\geq 30 \mathrm{~kg} / \mathrm{m}^{2}$ ) variou entre $15,4 \%$ e $23,4 \%$, nas cidades (MONTEIRO CA, 2020).

A obesidade é um fator de risco para várias condições crônicas. De acordo com a Sociedade Brasileira de Análises Clínicas (2018), no Brasil, cerca de 19,8\% da população é obesa, sendo que $80-90 \%$ desses pacientes possuem diabetes mellitus tipo 2 (DM2). Isso pode ter ocorrido devido ao fato de os adipócitos viscerais liberarem adipocinas pró-inflamatórias como IL- 6 e TNF- $\alpha$, leptina e fatores de crescimento que resultam em uma inflamação sistêmica que inibe a ativação do receptor da insulina, dificultando o transporte intracelular da glicose, causando resistência insulínica (LEITE LD, et al., 2009).

O diagnóstico da obesidade vai além do Índice de Massa Corporal (IMC), seu tratamento é complexo e deve ser conduzido, preferencialmente, de forma multidisciplinar. As terapias com o uso medicamentoso devem ser somadas com a modificação individualizada de hábitos de vida, sob supervisão médica contínua. 
Atualmente, para o tratamento de obesidade apenas três medicamentos são aprovados: sibutramina, orlistate e liraglutida. Apenas $10 \%$ dos pacientes com obesidade são diagnosticados e porcentagem inferior a $2 \%$ dos mesmos recebem tratamento para obesidade. Sabe-se que o tratamento clínico (mudança de estilo de vida e medicamentos) reduz em média 10\% do peso corporal (ABESO, 2016).

Esse artigo apresenta uma revisão do diagnóstico e tratamento da obesidade, com uma abordagem ampliada e multidisciplinar, demonstrando os resultados ao se integrar a Terapia Comportamental Intensiva para Obesidade (IBT) em conjunto com a farmacoterapia (Liraglutida) e mudanças no estilo de vida.

\section{REVISÃO BIBLIOGRÁFICA}

\section{Fisiopatogenia}

Entende-se que a obesidade é a soma de fatores genéticos e ambientais, uma vez que, para explicar o ganho de peso é necessário que exista um balanço energético positivo, ou seja, o indivíduo consome mais calorias do que gasta, no entanto, existe a influência genética, no que diz respeito ao risco de um indivíduo apresentar obesidade ao longo da vida. Estudos genômicos indicam uma predisposição genética à obesidade, já que foram identificadas mais de 140 regiões genéticas cromossômicas relacionadas à obesidade, entre eles alguns genes capazes de controlar a lipogênese e betaoxidação. Em relação aos fatores ambientais, tem-se a composição da dieta, com aumento das calorias ingeridas e redução das calorias gastas, fatores socioeconômicos, sedentarismo e tabagismo como fatores de influência na obesidade (GADDE KM, et al., 2018; SCHWARTZ MW, et al., 2017; SALES P, et al., 2016).

O sistema nervoso central tem a função de adequar a ingestão de alimentos do indivíduo às suas necessidades calóricas do momento, sendo assim, o cérebro utiliza informações vindas da periferia para identificar o nível de energia do organismo, essas informações são integradas no hipotálamo, que tem uma função importante na deteç̧ão da fome e organização do comportamento da alimentação. Além disso, essa capacidade do hipotálamo é integrada a outras regiões cerebrais como o córtex e o sistema límbico, que têm uma importante função no processamento de informações externas, como visão, olfato, paladar, memória e através do controle cognitivo, emocional e a tomada de decisões baseada em recompensas, já que o ser humano é capaz de se alimentar mesmo sem sentir fome, apenas pelo prazer (KISHORE M, et al., 2018; CARNELL, CS, et al., 2012; SALES P, et al., 2016).

\section{Consequências biopsicossociais da obesidade}

A obesidade é prejudicial à saúde, pois aumenta o risco de complicações e reduz a vida útil, acrescendo as taxas de morbimortalidade a nível mundial. As complicações ocorrem devido ao excesso, à localização e à distribuição da adiposidade e estão relacionados à interação dos fatores ambientais, genéticos, metabólicos, comportamentais e socioeconômicos (WHARTON MD, et al., 2020).

O distúrbio do peso corpóreo (obesidade) é fator de risco para diversas doenças crônicas não transmissíveis (DCNT), como DM2, doença da vesícula biliar, hepática gordurosa não alcoólica (DHGNA) e gota; além de contribuir para o aumento dos riscos cardiometabólicos, uma vez que facilita o desenvolvimento de doenças cardiovasculares (DCV) e diversos tipos de câncer (cólon, rim e esôfago - ambos sexos - e endométrio e mama nas mulheres) (WHARTON MD, et al., 2020).

Além do exposto, a maioria dos indivíduos obesos apresentam preconceito generalizado de peso e estigma, assim, afetam negativamente a saúde física e psicológica contribuindo para o desenvolvimento de quadros depressivos que dificultam os cuidados com a saúde, aumentando a prevalência e agravando o cenário e suas variáveis. Logo, a obesidade é vista como problema de saúde pública que ocasiona prejuízos individuais e coletivos, dado que aumenta os custos com cuidados de saúde (WHARTON MD, et al., 2020).

\section{Diagnóstico}

A diretriz Canadense de prática clínica propõe uma abordagem ampliada para avaliar a obesidade no adulto, na qual o diagnóstico começa reconhecendo a necessidade de aprofundar a avaliação clínica, por parte da pessoa e do médico (que deve pedir a permissão para tratar do assunto de forma empática e sem 
prejulgamentos). A partir de então, deve-se abordar diversos parâmetros na avaliação clínica: anamnese completa, exame físico, avaliação metabólica e psicológica, para aí então chegar ao tratamento ideal, em que o foco é a saúde integral da pessoa, a longo prazo (WHARTON MD, et al., 2020).

A avaliação física contempla os seguintes parâmetros recomendados (Grau: A Classe I ou Ila): Índice de Massa Corporal (IMC divisão do peso em $\mathrm{kg}$ pela altura em metros elevada ao quadrado, $\mathrm{kg} / \mathrm{m}^{2}$ ) e risco de doença crônica ou mortalidade: 25 a $29,9 \mathrm{~kg} / \mathrm{m}^{2}$ sobrepeso, risco pouco elevado; 30 a $34,9 \mathrm{~kg} / \mathrm{m}^{2}$ Obesidade grau I, risco elevado; 30 a $39,9 \mathrm{~kg} / \mathrm{m}^{2}$ obesidade grau II risco muito elevado; $\geq 40,0 \mathrm{~kg} / \mathrm{m}^{2}$ Obesidade grave grau III, risco muitíssimo elevado; a circunferência abdominal, que reflete a gordura visceral, se igual ou superior a $94 \mathrm{~cm}$ em homens e $80 \mathrm{~cm}$ em mulheres se correlaciona com o risco cardiovascular aumentado (ABESO, 2016).

Segundo o National Cholesterol Education Program (NCEP), o ponto de corte deve ser de $102 \mathrm{~cm}$ para homens e $88 \mathrm{~cm}$ para mulheres e deve-se considerar os ajustes segundo a idade e grupos étnicos; Relação cintura-estatura, com índice inferior a 0,5 , visto que valores superiores associam-se a maior risco de doenças cardiovasculares, DM2 e mortalidade por todas as causas; por fim, a análise de bioimpedância com medida da água corporal e da massa livre de gordura que, através de equações com peso, estatura e sexo, resulta na avaliação da composição corporal, cuja eficácia está bem estabelecida (ABESO, 2016).

A avaliação metabólica contempla a aferição da pressão arterial em ambos os braços e solicitação de exames complementares, conforme necessário, para avaliar comorbidades como a dislipidemia, o DM2, a presença de doença hepática e, assim, determinar o risco cardiometabólico. A saúde mental pode ser avaliada a partir de entrevista, com vistas a entender o contexto social e história abrangente do indivíduo. A partir desta avaliação é possível identificar ainda o comprometimento do bem-estar ou presença de sintomas de depressão, ansiedade, compulsão alimentar, bem como questões relacionadas à autoestima e identidade. (ABESO, 2016).

Questionários de saúde como o IWQOL-Lite e o SF36v2 podem ser úteis na avaliação dos parâmetros de qualidade de vida. Deve-se avaliar, ainda, o estado funcional com identificação de anormalidades ortopédicas e apneia do sono. Por fim, todas as informações podem analisadas usando o sistema de estadiamento da obesidade Edmonton Obesity Staging System (EOSS) que classifica a obesidade em 5 estágios, considerando os parâmetros apresentados de forma a auxiliar o delineamento do tratamento ideal para o indivíduo com obesidade (WHARTON MD, et al., 2020; KOLOTKIN RL, et al., 2017).

\section{Tratamento Clínico}

Lidar com a obesidade de forma eficaz requer uma abordagem multidisciplinar e integrada, que vai além do indivíduo (biológico, psicológico) e considera fatores sociais (ambiente, socioeconômico, cultural, educação) que determinam os resultados em diversos níveis de saúde (individual, comunitário e populacional). Essa abordagem leva ao reconhecimento da complexidade do tema obesidade e outras questões que envolvem saúde pública (VANDENBROECK P, et al., 2007; MCGLASHAN J, et al., 2018).

Individualmente, é necessário definir metas de forma colaborativa, acompanhar o paciente (reavaliar e melhorar o tratamento), ampliar opções, bem como discutir as principais opções de tratamentos e terapias adjuvantes que podem ser necessárias, incluindo intervenções psicológicas, farmacológicas e cirúrgicas. Todas essas condutas devem ser em concordância com o paciente, com foco nos benefícios e nos ganhos a longo prazo (WHARTON MD, et al., 2020).

Além do exposto, é indispensável o apoio dos profissionais de saúde à pessoa e o incentivo à melhoria do atendimento para essa doença crônica em diversos âmbitos do sistema de saúde (WHARTON MD, et al., 2020).

\section{Tratamento medicamentoso}

O peptídeo semelhante ao glucagon 1 (GLP-1) é uma substância secretada pelas células enteroendócrinas do trato intestinal que atua sobre os processos que envolvem a digestão e o metabolismo. Ele atua no aumento da secreção de insulina induzida pela glicose, na inibição da gliconeogênese e na secreção de 
glucagon e retardo do esvaziamento gástrico, atuando, assim, a nível central sobre os mecanismos de apetite e saciedade no hipotálamo. Sua meia-vida é relativamente curta (cerca de dois minutos) e sua degradação é feita pela enzima dipeptidil-peptidase IV (DPP-IV). Devido a essa importante influência sobre a glicemia e o apetite, o GLP-1 passou a ser amplamente estudado no contexto da obesidade e do DM2 - o que motivou o desenvolvimento de fármacos análogos a essa substância com o objetivo de modular seus efeitos no organismo e obter avanços no tratamento dessas comorbidades (NAUCK M, 2016; DRUCKER DJ, 2018).

Os medicamentos análogos ao GLP-1 buscam reproduzir os efeitos do GLP-1 endógeno de forma mais eficaz, já que serão menos suscetíveis à degradação pela enzima DPP-IV, com aumento do tempo de ação e maximização dos efeitos desejados (NAUCK M, 2016; GENTILELLA R, et al., 2018). De acordo com Drucker DJ (2018), os principais efeitos adversos dos fármacos análogos ao GLP-1 são sintomas gastrointestinais como náuseas, vômitos e diarreia. Estes podem ser explicados pela ação inibitória da motilidade gastrointestinal provocada por esses medicamentos, culminando em um retardo do esvaziamento gástrico e, assim, na instalação dos sintomas.

Dentre os fármacos análogos ao GLP-1 existentes, a liraglutida (Saxenda® e Victoza®) é uma das substâncias mais conhecidas e amplamente utilizadas para o tratamento da obesidade, autorizada para uso no Brasil (ABESO, 2016). A liraglutida foi comprovada em estudo randomizado SCALE, que com dieta e exercícios, manteve a perda de peso alcançada pela restrição calórica e induziu maior perda de peso ao longo de 56 semanas. Também foram observadas melhorias em alguns fatores de risco para doenças cardiovasculares (WADDEN TA, et al., 2013).

A liraglutida possui uma meia-vida de cerca de 13 horas, sendo o pico de absorção 11 horas após administração subcutânea, fator substancial na maximização de seus efeitos desejados. Segundo a ABESO (2016) é classificada em grau A Classe I (forte) na escala de recomendação de fármacos empregados no tratamento da obesidade e sobrepeso no Brasil. (ABESO, 2016; PEREZ-MONTES DE OCA A, et al., 2020; NAUCK MA, et al., 2020; BLACKMAN A, et al., 2016).

Essa recomendação se dá, sobretudo, em casos onde houve falha no tratamento não-farmacológico em pacientes que preencham alguns requisitos, como a) $I M C \geq 30 \mathrm{~kg} / \mathrm{m}^{2}$; b) $I \mathrm{IMC} \geq 25 \mathrm{~kg} / \mathrm{m}^{2}$ e com pelo menos outra comorbidade associada (DM2, hipertensão arterial, hiperlipidemia, apneia do sono, entre outras); c) circunferência abdominal maior ou igual a $102 \mathrm{~cm}$ (homens) e $88 \mathrm{~cm}$ (mulheres). Em pacientes com esse perfil, as evidências demonstraram que o uso da liraglutida levou à melhora dos parâmetros glicêmicos, da pressão arterial, do perfil lipídico, de marcadores de risco cardiovascular e da qualidade de vida, além de melhora na apneia do sono (ABESO, 2016; PEREZ-MONTES DE OCA A, et al., 2020; NAUCK MA, et al., 2020; BLACKMAN A, et al., 2016).

Contudo, apesar de todos os seus benefícios no tratamento da obesidade e doenças associadas, a liraglutida é contraindicada em casos de gravidez. Outrossim, a eficácia e a segurança dessa substância não são bem descritas em pacientes portadores de insuficiência cardíaca, renal e hepática graves, o que faz com que uso seja vedado a esses indivíduos. Também, é contraindicada em pacientes que possuem gastroparesia, em virtude de seus efeitos inibitórios sobre a motilidade gastrointestinal. Por fim, outros fatores que podem limitar sua prescrição e adesão do paciente ao tratamento com a liraglutida são seus efeitos adversos sobre o trato gastrointestinal e o alto custo característico do tratamento (GADDE KM e ATKINS KD, 2020; ABESO, 2016; HERNÁNDEZ-RODRIGUEZ, 2019).

\section{Tratamento cirúrgico}

A cirurgia bariátrica (CB) é considerada, atualmente, como a terapia mais eficaz para o tratamento da obesidade grave, sendo destinada a redução de peso em pacientes entre 18 a 65 anos, com IMC superior a $40 \mathrm{~kg} / \mathrm{m}^{2}$ ou $35 \mathrm{~kg} / \mathrm{m}^{2}$ associado a uma ou mais comorbidades como DM2, HAS, dislipidemia, além de falha do tratamento clínico, que inclui mudança dos hábitos alimentares, prática de atividades físicas, acompanhamento psicológico e farmacoterapia, documentada por um período de, pelo menos, 2 anos. É um procedimento capaz de reduzir os índices de mortalidade e melhora das demais doenças que acometem os indivíduos, com restabelecimento do seu estado psicossocial e da qualidade de vida (ABESO, 2016; ANGRISANI L, et al., 2015). 
Além disso, a cirurgia pode ser realizada por dois tipos de técnicas, as não derivativas, que inclui banda gástrica laparoscópica ajustável e gastrectomia vertical e as derivativas, que são bypass gástrico em $Y$ de Roux e derivações biliopancreáticas com switch duodenal. Tais procedimentos atuam alterando a comunicação entre o trato gastrointestinal e neurocircuitos de homeostase energética referidos como "eixo intestino-cérebro" (ABESO, 2016; KISHORE MG, et al., 2018; SCHWARTZ MW, et al., 2017).

Somado a isso, há também a influência de hormônios gástricos, como a grelina; hormônios intestinais, como o peptídeo semelhante ao glucagon 1 e alterações no nível e composição de ácidos biliares e ou microbioma intestinal, que irão interferir no apetite do paciente, contribuindo para o emagrecimento. A perda ponderal após a CB, aliada à Terapia Comportamental Intensiva para Obesidade, atinge um pico por volta de 12 a 18 meses de pós-operatório, e de acordo com a técnica, pode variar de $14 \%$ a $43 \%$ no primeiro ano (ABESO, 2016; KISHORE MG, et al., 2018; SCHWARTZ MW, et al., 2017).

\section{Importância da abordagem multidisciplinar}

Estudos randomizados e exploratórios, explanados a seguir, demonstram a eficácia da Terapia Comportamental Intensiva para Obesidade (IBT), em conjunto com a farmacoterapia, com resultados clínicos significativos a curto e longo prazo.

Estudo randomizado feito por Wadden TA, et al. (2019) avaliou a eficácia da IBT associada a liraglutida, observou resultados favoráveis a essa abordagem, destacando aspectos como a realização de vinte e uma sessões semanais de IBT, seguido por visitas a cada duas semanas, com enfoque no aconselhamento de estilo de vida, realizada por profissionais de cuidados primários (PCPs), incluindo médicos e enfermeiros. Contribui para a perda de peso clinicamente significativa em um ano (redução média de 6,1\%) e a abordagem, com a adição de liraglutida à IBT, quase dobrou a perda média de peso $(11,5 \%)$. Isso porque houve alto envolvimento dos participantes e melhores taxas de retenção e adesão aos objetivos;

Por fim, os resultados dos grupos da pesquisa (IBT-sozinho, IBT-liraglutida e Multi-componente) foram: o grupo IBT-liraglutida obteve resultados superiores e mais duradouros que os demais grupos. A perda de peso em todos os três grupos foi associada a melhorias clinicamente significativas nos fatores de risco cardiometabólicos, são eles: pressão arterial sistólica e diastólica, frequência cardíaca, colesterol de lipoproteína de baixa densidade (LDL), triglicerídeos e depressão. Porém, houve melhorias significativamente maiores em um ou ambos grupos tratados com liraglutida, nos seguintes itens: lipoproteína de alta densidade (HDL), proteína $C$ reativa, glicose em jejum, hemoglobina A1c e saúde mental. A perda de peso, foi um contribuinte significativo para o efeito geral do tratamento (WADDEN TA, et al., 2019).

$O$ efeito do tratamento IBT e medicamento (Liraglutida) foi associado a benefícios no curto prazo no que diz respeito à desinibição alimentar, psicopatologia do transtorno alimentar global, além de mudanças na qualidade de vida relacionadas à saúde geral e relacionada ao peso. As melhorias a longo prazo foram observadas nos casos em que houve, além do exposto, a reeducação alimentar e exercícios físicos, o que possibilitou a manutenção dos benefícios verificados em três anos. Observou-se ainda melhorias em aspectos do funcionamento psicossocial, vitalidade e saúde mental. A qualidade de vida relacionada à saúde (QVRS), foi avaliada usando os questionários de saúde IWQOL-Lite e o SF36v2. Estudos adicionais são necessários para investigar os mecanismos subjacentes às perdas de peso a longo prazo, observadas com liraglutida (CHAO AM, et al., 2010; CHAO AM, et al., 2019; KOLOTKIN RL, et al., 2017).

A respeito da abordagem multidisciplinar em pacientes que cumprem os requisitos e que optaram por CB, o acompanhamento longitudinal após a cirurgia é primordial para o sucesso de tal terapia. O seguimento por meio de consultas e exames laboratoriais deve ser regular, a fim de identificar precocemente possíveis complicações metabólicas e nutricionais. $O$ paciente deve ser orientado quanto a sua nova dieta e a necessidade de suplementação multivitamínica e mineral. Aliado a tais medidas, a Terapia Comportamental Intensiva para Obesidade é fundamental para a maior eficácia do tratamento ao estimular a autoestima do indivíduo, segurança com a mudança corporal, prática de exercícios físicos, contribuindo para melhora da qualidade de vida (ABESO, 2016; MELO WV, et al., 2014).

Esses resultados têm implicações importantes para o manejo da obesidade na prática da atenção primária, pois fornece aos médicos a confiança para orientar os pacientes sobre os benefícios da perda de peso com 
foco na melhoria da qualidade de vida. Mostram, ainda, um caminho possível que exige capacitação, recursos e integração da equipe de atendimento para oferecer amplo acesso a um tratamento adequado, com resultados clinicamente satisfatórios. Reconhecidamente, no Brasil, o sobrepeso associado a doenças e a obesidade fazem parte da área de atuação do endocrinologista, porém devido à sua elevada prevalência, é importante que outras especialidades médicas, principalmente os que atuam na atenção básica, sejam capacitados para reconhecer e encaminhar os pacientes oportunamente e até mesmo a tratar os pacientes (CHAO AM, et al., 2019; WADDEN TA, et al.,2019; ABESO, 2016).

\section{CONSIDERAÇÕES FINAIS}

Por meio deste estudo de revisão bibliográfica, corrobora-se a importância de uma abordagem multidisciplinar no tratamento da obesidade, de forma a abordar o tema com empatia, definindo metas de forma colaborativa e com acompanhamento. Observou-se que a liraglutida aliada à Terapia Comportamental Intensiva (IBT) é eficaz no tratamento da obesidade, auxilia no controle dos valores glicêmicos, e é uma alternativa para pacientes com DM2. Contudo, apesar dos excelentes resultados em pacientes em uso de liraglutida, apresenta como efeitos adversos náuseas, vômitos e diarreia devido um retardo no esvaziamento gástrico. Dessa maneira, a liraglutida é capaz de desenvolver perda de peso significativa e melhora no comportamento alimentar, porém deve ser usada a partir de uma recomendação médica.

\section{REFERÊNCIAS}

1. ANGRISANI L, et al. Bariatric Surgery Worldwide 2013. Obes Surg, 2015; 25:1822-1832.

2. ASSOCIAÇÃO BRASILEIRA PARA O ESTUDO DA OBESIDADE E DA SÍNDROME METABÓLICA (ABESO). Diretrizes brasileiras de obesidade. 4. ed. São Paulo: ABESO, 2016; 7-150.

3. BLACKMAN A, et al. Effect of liraglutide $3.0 \mathrm{mg}$ in individuals with obesity and moderate or severe obstructive sleep apnea: the SCALE Sleep Apnea randomized clinical trial. Int J Obes (Lond), 2016; 40(8): 1310-9.

4. CARNELL CS, et al. Neuroimaging and obesity: current knowledge and future directions Obes Rev, 2012; 13: 43-56.

5. CHAO AM, et al. Changes in health-related quality of life with intensive behavioural therapy combined with liraglutide $3.0 \mathrm{mg}$ per day. Clinical Obesity, 2019; 9(6): e12340.

6. CHAO AM, et al. Effects of Liraglutide and Behavioral Weight Loss on Food Cravings, Eating Behaviors, and Eating Disorder Psychopathology. Obesity (Silver Spring), 2019; 27(12): 2005-2010.

7. DRUCKER DJ. Mechanisms of Action and Therapeutic Application of Glucagon-like Peptide-1. Cell Metabolism, 2018; 27(4): 740-756.

8. GADDE KM, ATKINS KD. The limits and challenges of antiobesity pharmacotherapy. Expert Opinion on Pharmacotherapy, 2020; 21(11): 1319-1328.

9. GADDE KM, et al. Fisiopatologia e Manejo da Obesidade. Journal of the American College of Cardiology, 2018; 71(1): 1-16.

10. GENTILELLA R, et al. Glucagon-like peptide-1 receptor agonists in type 2 diabetes treatment: are they all the same?. Diabetes Metab Res Rev, 2018; 35(1): e3070.

11. KISHORE MG, et al. Obesidade: Fisiopatologia e Manejo. Journal of the American College of Cardiology, 2018; 71(1): 69-84.

12. KOLOTKIN RL, et. al. Improvements in health-related quality of life over 3 years with liraglutide $3.0 \mathrm{mg}$ compared with placebo in participants with overweight or obesity. Clinical Obesity, 2018; 8(1): 1-10.

13. HERNÁNDEZ-RODRÍGUEZ DC. Eficacia y seguridad de liraglutida como tratamiento coadyuvante para disminuir el índice de masa corporal. Rev Salud Bosque, 2019; 9(2): 47-55.

14. LEITE LD, et al. Obesidade: uma doença inflamatória. Ciência \& Saúde, 2009; 2: 85-95.

15. MCGLASHAN J, et al. Comparando perspectivas complexas sobre os impulsionadores da obesidade: comunidades voltadas para a ação e especialistas orientados por evidências. Obes Sci Pract, 2018; 4(6): 575-581.

16. MELO WV, et al. A terapia cognitivo-comportamental e a cirurgia bariátrica como tratamentos para a obesidade. Revista Brasileira de Terapias Cognitivas, 2014;10(2):84-92.

17. MONTEIRO CA, et al. Vigitel Brasil 2019: vigilância de fatores de risco e proteção para doenças crônicas por inquérito telefônico: estimativas sobre frequência e distribuição sociodemográfica de fatores de risco e proteção para doenças crônicas nas capitais dos 26 estados brasileiros e no Distrito Federal em 2019. Brasília: Ministério da Saúde, 2020; 35-42.

18. MORETZSOHN M, et al. Nutrologia. Sociedade de Pediatria do Estado do Rio de Janeiro, 2015; 184.

19. NAUCK MA, et al. GLP-1 receptor agonists in the treatment of type 2 diabetes - state-of-the-art. Molecular Metabolism, 2020; 14: e101102.

20. PEREZ-MONTES DE OCA A, et al. Obesity and GLP-1. Minerva Endocrinology, 2020; 19.

21. SALES $P$, et al. O essencial em endocrinologia. - 1. ed. - Rio de Janeiro: Roca, 2016; 816.

22. SCHWARTZ MW, et al. Obesity Pathogenesis: An Endocrine Society Scientific Statement. Endocr Rev, 2017; 38(4): $267-296$.

23. SOCIEDADE BRASILEIRA DE ANÁLISES CLÍNICAS, 2018. Brasil: SBAC. Disponível em: www.sbac.org.br. Acesso em: 22 nov. 2020.

24. VANDENBROECK PI, et al. Tackling Obesities: Future Choices - Obesity System Atlas. Government Office for Science London, 2007; 1-46.

25. WADDEN TA, et al. Intensive Behavioral Therapy for Obesity Combined with Liraglutide 3.0 mg: A Randomized Controlled Trial. Obesity (Silver Spring), 2019; 27(1): 75-86.

26. WADDEN TA, et al. Weight maintenance and additional weight loss with liraglutide after low-calorie-diet-induced weight loss: the SCALE Maintenance randomized study. International journal of obesity, 2013; 37(11): 1443-1451.

27. WHARTON MD, et. al. Obesity in adults: a clinical practice guideline. Canadian Medical Association Journal, 2020. 